\title{
Communication évaluée dans le service d'assistance téléphonique
}

\section{Magdalena Maj}

\section{(2) OpenEdition}

1 Journals

Édition électronique

URL : http://journals.openedition.org/communicationorganisation/1430

DOI : 10.4000/communicationorganisation. 1430

ISSN : $1775-3546$

Éditeur

Presses universitaires de Bordeaux

\section{Édition imprimée}

Date de publication : 1 décembre 2010

Pagination : 101-108

ISBN : 978-2-86781-743-4

ISSN : $1168-5549$

\section{Référence électronique}

Magdalena Maj, «Communication évaluée dans le service d'assistance téléphonique », Communication et organisation [En ligne], 38 | 2010, mis en ligne le 01 décembre 2013, consulté le 19 avril 2019. URL: http://journals.openedition.org/communicationorganisation/1430 ; DOI : 10.4000/ communicationorganisation. 1430 


\title{
Communication évaluée dans le service d'assistance téléphonique
}

\author{
Magdalena MAJ'
}

\section{Introduction}

«On ne peut pas ne pas communiquer» constate l'un des axiomes de la communication selon Watzlawick. L'avènement d'une société fondée sur la communication, d'une moderne société de contact a fait en effet ouvrir le débat non plus sur « le pourquoi communiquer? " mais déjà sur le "comment communiquer?" [Westphalen, 1997, p. 6]. Limpératif de "l'organisation communicante » et celui de «la communication organisée » [Bartoli, 1991, p. 102] ne laissent guère de doute. Suivant cette logique, les organisations contemporaines qui fonctionnent sur des marchés supranationaux et de plus en plus compétitifs se voient dans l'obligation d'établir un plan d'action cohérent et logique que l'on appelle une stratégie de communication. Porteuse des traits du discours organisationnel et produite au terme d'un processus collectif souvent long et complexe, la stratégie de communication et son élaboration engagent une bonne partie de ressources humaines, temporelles et financières d'organisations commerciales et économiques d'aujourd'hui.

Dans cet article nous allons présenter les principales composantes de la stratégie de communication qu'une compagnie aérienne a mise en place au sein de son service d'assistance téléphonique, parmi lesquelles les mécanismes d'évaluation et de contrôle de la qualité tiennent une place extrêmement importante. Cette recherche tente de montrer les relations complexes qui se sont nouées entre évaluation et communication en tant que deux éléments interdépendants et corrélatifs: sans évaluation rigoureuse et objective il n'y a pas de communication efficace.

Notre étude se base sur des données et documents authentiques collectés dans le centre d'appels d'une compagnie aérienne américaine où la communication est exolingue car elle se fait entre locuteurs qui ne possèdent pas la même

1. Magdalena Maj est doctorante à I'Institut de Philologie Romane à I'Université Adam Mickiewicz de Poznan. Son travail de recherche porte sur le discours organisationnel construit autour de la relation au client dans le contexte du service d'assistance téléphonique. Mail : mayma@wp.pl 
langue maternelle: clients francophones et agents d'origine étrangère. Ces données ont été recueillies en l'espace de presque deux ans (2009-10) sous forme de documents professionnels que la compagnie adresse à son personnel et sous forme d'enregistrements des appels téléphoniques (pratique usuelle dans plusieurs établissements de ce type).

\section{Communication et évaluation}

Vague et utilisée souvent dans des sens très différents, la notion de communication nécessite ici une définition précise et un positionnement épistémologique et méthodologique sans ambiguité. Dans notre recherche nous l'abordons selon une perspective linguistique, interactionniste et discursive. De cette manière et au sens traditionnel du terme, la communication c'est tout échange verbal et non verbal ayant lieu entre au moins deux participants, parfois appelés acteurs de la communication. La situation de communication peut être définie par la formule « je, ici, maintenant », «je » symbolisant les participants à la communication et « ici, maintenant » se référant au cadre spatio-temporel de l'énoncé ou au contexte situationnel.

La dimension interactionniste de la communication consiste en son caractère dynamique: une influence mutuelle et constante des acteurs, « une activité collective de production du sens, activité qui implique la mise en œuvre de négociations explicites ou implicites, qui peuvent aboutir, ou échouer » [Kerbrat-Orecchioni, 1995, p. 28-29]. La communication ne s'associe donc pas - ou pas seulement - à l'information car "l'information vaut et se mesure dans le champ de la connaissance et la communication dans celui de l'action et de l'organisation. De ce partage découle que la seconde précède et conditionne nécessairement la première » [Bougnoux, 1998, p. 72].

L'approche discursive de la communication telle que nous l'analysons, veut dire qu'elle est représentative d'un certain type de discours, notamment discours organisationnel ou institutionnel, dont deux manifestations ont été définies en tant que récits d'entreprise [D'Almeida, 2001, p. 97-98], une certaine mythologie au service de grandes organisations commerciales visant à leur redonner une dimension humaine et sociale. Ces deux points de vue, interactionniste et discursif, convergent dans la mesure où les interactions qui se tiennent entre clients et agents du service d'assistance téléphonique de l'entreprise faisant l'objet de notre étude, portent les traits du discours institutionnel, et comme telles subissent une évaluation organisationnelle permanente.

Effectivement, la communication telle que nous venons de la définir et l'évaluation qui la suit dans le processus de la gestion de la qualité constituent deux étapes consécutives et indissociables. Nous utilisons le terme d'évaluation dans son acception de «jugement de valeur» porté sur les pratiques, "par référence à des critères et à des normes explicites (ex. sa pertinence, son efficience) » [Commission européenne, 1999, p. 17], critères 
et normes d'appréciation qualitative qui ont été élaborés dans les documents professionnels qu'une entreprise multinationale adresse aux agents du service d'assistance téléphonique. Les mécanismes d'évaluation permettent ainsi d'apprécier les résultats au regard d'objectifs fixés. L'évaluation des pratiques communicatives et professionnelles fondée sur une méthode rigoureuse, s'inscrit désormais dans l'idéologie de nombreuses organisations contemporaines, notamment celles de services aux personnes, et en tant que composante cruciale, fait partie d'une stratégie de communication.

\section{Stratégie de communication}

Nous tenons à insister en ce lieu sur la notion de stratégie définie comme un « ensemble d'actions coordonnées, d'opérations habiles, de manœuvres en vue d'atteindre un but précis " (Trésor de la Langue Française informatisé). Cela converge avec l'idée de la communication organisationnelle qui tend à réfuter "l'illusion de la spontanéité ", "le sentiment tenace de l'évidence et de la facilité » des processus d'organisation et de communication [Bartoli, 1991, p. 99]. Dans cette perspective, tous les choix relatifs à une stratégie de communication visent à établir une relation cohérente entre les éléments constitutifs de cette stratégie, où les mécanismes d'évaluation occupent une place prépondérante.

Lanalyse de la politique de communication de la compagnie, centrée notamment sur ses relations avec les clients au sein du service d'assistance téléphonique, nous permet d'en dégager quelques composantes importantes:

1. Les sociétés de conseil qui à la base de nombreuses analyses, recherches et enquêtes (outils psycho- et sociologiques), formulent des consignes et recommandations en vue d'améliorer la communication avec les clients de la compagnie et d'optimiser le processus d'évaluation de manière à le rendre plus fiable et pertinente;

2. La formation des agents: les agents suivent une formation professionnelle de six semaines. Ils sont ensuite continûment formés en communication fluide et efficace (clients difficiles, situations conflictuelles, vocabulaire préconisé, ouverture et clôture de l'appel);

3. Les ressources internes: les agents font usage de tout un éventail de ressources informatisées accessibles depuis le monde entier par tous les départements et agents connectés. Il s'agit notamment d'indices techniques mais aussi de consignes de communication verbale et vocale en vue de satisfaire le client;

4. Mécanismes d'évaluation et de contrôle: tous les appels sont enregistrés et réécoutés pour des buts de contrôle de la qualité. Les appels sont ensuite évalués, principalement sous l'angle de leur conformité avec les consignes de communication réussie et efficace délivrées par la compagnie. Les résultats de cette évaluation influencent ensuite les rémunérations des agents donc font partie d'un dispositif de motivation financière. 


\section{Méthodes d'évaluation interne}

Toute stratégie de communication cohérente et bien conçue prévoit donc certaines pratiques évaluatives susceptibles de vérifier sa pertinence et efficacité. Dans ce qui suit, nous proposons une analyse plus poussée de mécanismes d'évaluation mis en place par la compagnie qui constitue l'objet de cette étude.

L'enregistrement et la réécoute des appels téléphoniques permettent de voir si les comportements langagiers et autres (pratiques communicatives effectives) des agents sont en correspondance avec la stratégie de communication de la compagnie (pratiques communicatives normatives). Codifiés, ces comportements imposés aux agents laissent très peu de place aux libres choix et spontanéité d'énoncés. Certains des appels enregistrés sont sélectionnés sur des critères aléatoires, réécoutés et évalués indépendamment par deux personnes, supérieur hiérarchique direct d'un agent et superviseur externe, dont les évaluations sont ensuite confrontées en vue de vérification de leur justesse et objectivité. Les résultats d'une telle évaluation qui s'effectue une fois par semaine, sont présentés aux agents.

La fiche d'évaluation est très détaillée et développée. Elle comporte d'abord des indications générales sur date, heure et lieu de l'entretien téléphonique, sa durée et son caractère (requête générale ou demande de renseignements particuliers, demande de service, transaction commerciale), nom d'une personne évaluée et de celle qui évalue, ainsi que date de l'évaluation. Tous ces moyens servent à donner à l'évaluation les traits d'un processus sérieux, méticuleux, méthodique et rigoureux.

Axée sur les attitudes et comportements professionnels des agents, la fiche d'évaluation présente trois sections: comportements cruciaux du point de vue des clients, comportements cruciaux pour la compagnie même, et enfin comportements procéduraux. Les deux dernières sections imposant des critères économiques et réglementaires (compétence de vente, respect de politiques et procédures de la compagnie, protection d'informations personnelles des clients), nous nous concentrerons sur la première, la plus développée, qui occupe une place centrale dans le processus d'évaluation de la communication orientée vers les besoins du client. Cette section évalue les pratiques communicatives et professionnelles employées par les agents, notamment celles qui portent directement sur la satisfaction client et son expérience positive ou bien négative. Elle cherche alors à se mettre dans une position de clients et à évaluer la qualité du service client de sa perspective. Cette section contient alors des critères particuliers relatifs à:

a. solution d'un problème proposée/réponse pertinente apportée

b. degrés de politesse présentée par l'agent

c. compétence de communication linguistique

d. volonté d'aide affichée par l'agent

e. gestion du temps et contrôle de la conversation 
f. renseignements précis et exhaustifs fournis

g. tenue d'un dossier client (est-il clair et uniforme)

h. récapitulation des points principaux de la requête.

Chacun des critères sus mentionnés apportent de nombreux exemples de comportements non respectueux des principes d'une communication efficace et harmonieuse. La violation d'un des points cruciaux de la fiche d'évaluation est considérée comme une atteinte portée à un bon service client.

La rigueur et la précision avec lesquelles l'évaluation est menée au sein du service d'assistance téléphonique témoignent de l'importance que les organisations contemporaines accordent aux normes d'appréciation qualitative dans des secteurs professionnels de services aux personnes (clients). Une véritable méthode d'évaluation, conséquente et consistante, rend possible la comparabilité des résultats et permet ainsi d'éliminer les comportements jugés comme non désirables, et de renforcer ceux qui sont considérés comme souhaitables du point de vue de l'intérêt d'une organisation, promouvant de cette manière une image cohérente et logique de la compagnie et de sa stratégie de communication.

\section{Procédés d'évaluation externe}

Jusqu'à présent nous n'avons parlé que des mécanismes d'évaluation interne, c'est-à-dire celle qui s'accomplit au sein de la compagnie et suivant ses critères organisationnels. Non moins importante, sinon plus, est l'évaluation faite par les clients qui s'effectue à travers le questionnaire de satisfaction client. Cette idée s'inscrit dans la logique de la gestion de la qualité qui attribue une place importante à la voix du client, "une communication permanente » et «structurée » [Gogue, 1993, p. 94] entre l'organisation et son marché, représenté bien évidemment par ses clients.

Nous nous proposons en ce lieu d'analyser la construction du questionnaire de satisfaction client et de présenter les difficultés qu'il engendre dans certaines situations communicatives.

En fin de chaque appel et ceci sans exception, les agents se trouvent dans une obligation contraignante de proposer aux clients une enquête qui porte sur cette concrète conversation téléphonique. Les clients ont donc la possibilité de s'exprimer sur la qualité du service rendu par les représentants de la compagnie en répondant aux questions suivantes:

1. Êtes-vous satisfait de l'amabilité de notre représentant?

2. Vous êtes très satisfait, tapez 5. Satisfait, tapez 4. Moyennement satisfait, tapez 3. Pas satisfait, tapez 2. Très insatisfait, tapez 1.

3. Etes-vous satisfait de la façon avec laquelle vous avez pu communiquer avec notre représentant?

4. Etes-vous satisfait de la volonté de vous assister de notre représentant?

5. Etes-vous satisfait du temps mis pour répondre à votre demande? 


\section{Notre représentant a-t-il été en mesure de répondre entièrement à votre demande?}

7. Oui, tapez 1. Non, tapez 2.

8. Êtes-vous satisfait de l'ensemble du service rendu par notre service réservations aujourd'bui?

9. Vous êtes très satisfait, tapez 5. Satisfait, tapez 4. Moyennement satisfait, tapez 3. Pas satisfait, tapez 2. Très insatisfait, tapez 1.

L'enquête de satisfaction client est obligatoirement proposée à tous les clients individuels et sans tenir compte de l'objet de l'appel ni de son résultat. Cela conduit à des situations involontairement incommodes tant pour l'agent que le client où la moindre suggestion concernant le questionnaire de satisfaction semble incongrue et déplacée. Nous pensons notamment à des situations délicates ou conflictuelles dues à certains torts causés aux clients par la compagnie et ses représentants, certainement sans intention de nuire, mais qui portent quand même atteinte à l'image de l'entreprise. Il y aurait aussi celles encore où le motif de l'appel étant bref et anodin, le client se serait contenté d'une réponse concise et rapide mais la contrainte évoquée en supra pousse l'agent à prolonger inutilement la conversation en essayant de le faire répondre aux questions. Sans enfin parler d'absurdités évidentes comme proposer une enquête à une personne qui s'est juste trompé de numéro.

Á cela s'ajoute encore le problème que pose la construction même du questionnaire où nous pouvons remarquer à un certain moment (questions quatre à six) une inversion de l'ordre logique de réponses ce qui risque, et c'est souvent le cas, de désorienter la personne interrogée. Tout de même une telle succession (il)logique de questions est entièrement délibérée et vise apparemment à vérifier tant la vigilance du client que la fiabilité des résultats obtenus.

L'évaluation effectuée par les clients pose un problème majeur lié à la question suivante: dans quelle mesure est-elle gérable tant par la compagnie toute entière que par ses agents? Dépend-elle réellement des compétences professionnelles, communicatives et linguistiques des agents? Le critère de satisfaction étant subjectif et difficilement mesurable, les résultats de l'évaluation externe restent toujours un facteur hautement imprévisible. D'autant plus que fréquemment les intérêts respectifs de la compagnie et de ses clients divergent.

\section{Conclusion}

Les organisations de services aux personnes ont connu ces derniers temps un développement rapide des mécanismes d'évaluation des pratiques de travail. Adressant leurs activités professionnelles au grand public, elles s'appliquent à écouter la voix des clients sans pour autant perdre de vue leurs objectifs économiques. 
Nous avons proposé dans cet article l'étude de la stratégie de communication d'une compagnie aérienne et du processus d'évaluation qu'elle a mis en place au regard des agents de son service d'assistance téléphonique. Dans ce type de service (centre d'appels) l'évaluation porte sur les compétences professionnelles des agents du service client, avec l'accent mis sur la façon dont ils gèrent les interactions verbales avec les passagers. Les résultats de leur travail sont soumis à une évaluation multi-étapes, très développée et croisée car confrontant deux axes principaux et points de vue parfois divergents: évaluation interne menée par les services de contrôle de la qualité et évaluation externe effectuée par les clients. Si la première se laisse définir par des critères suffisamment clairs afin de permettre la gestion des effets de communication, la seconde semble échapper au contrôle des acteurs de communication.

Par ailleurs, la confrontation des résultats de ces deux évaluations indépendantes mais portant sur la même conversation téléphonique peut montrer la différence des perspectives et mettre en lumière les divergences soit des intérêts respectifs de la compagnie et ses clients, soit leur conception distincte du but social des interactions de service qui est celui de satisfaction client. Nous pensons notamment à des situations où l'agent ne peut pas répondre favorablement aux sollicitations du client car celles-ci vont à l'encontre des politiques et procédures, c'est-à-dire certains standards qui sont inviolables. L'objectif de la satisfaction client reste ainsi insatisfait.

Cela nous conduit enfin à l'interrogation sur des relations complexes entre évaluation d'un côté et communication, compétence et norme de l'autre. Quels sont, dans le contexte du service client, les normes et les standards de satisfaction client? Comment les définir et mesurer? Quels sont les facteurs qui influencent la satisfaction client? Ne dépend-elle que des compétences professionnelles des agents du service client? Telles sont les questions auxquelles cet article a voulu se confronter, avec toute conscience quand même que dans le secteur des services aux personnes il n'existe pas de communication efficace et cohérente sans évaluation méthodique et organisée.

\section{BIBLIOGRAPHIE}

BARTOLI Annie. Communication et organisation. Pour une politique générale cohérente. Paris: Les Éditions d'Organisation, 1991, 175 p.

BOUGNOUX Daniel. Introduction aux sciences de la communication. Paris: Éditions La Découverte, 1998, $125 \mathrm{p}$.

COMMISSION EUROPÉENNE. Évaluer les programmes socio-économiques. Glossaire de 300 concepts et termes techniques. Vol.6. Luxembourg: Office des publications officielles des Communautés européennes, 1999, 138 p.

D'ALMEIDA Nicole. Les promesses de la communication. Paris: Presses Universitaires de France, 2001, 264 p. 
GOGUE Jean-Marie. Management de la Qualité. Paris: Economica, 1993, 110 p. KERBRAT-ORECCHIONI Catherine. Les Interactions verbales. Tome I. $2^{\mathrm{e}}$ éd. Paris: Armand Colin, 1995, 318 p.

WESTPHALEN Marie-Hélène. La communication externe de l'entreprise. Paris: Dunod, 1997, 128 p.

Résumé : L'impératif de la communication organisée contraint les organisations de services aux personnes à mettre en place des stratégies de communication cohérentes qui contribuent à créer une image homogène de l'organisation. L'efficacité des pratiques communicatives instaurées par l'organisation est constamment suivie grâce au dispositif d'évaluation très développé. En prenant appui sur l'exemple d'une compagnie aérienne d'origine nord-américaine, cet article analyse les méthodes d'évaluation interne (effectuée par les services de contrôle de qualité) et externe (effectuée par les clients) mises en œuvre au sein de son service d'assistance téléphonique. La comparaison des résultats de ces deux évaluations indépendantes mais portant sur la même conversation téléphonique peut montrer la divergence soit des intérêts respectifs de la compagnie et de ses clients, soit leur conception distincte du but social des interactions de service qu'est la satisfaction du client.

Mots clés: interactions de service, satisfaction client, stratégie de communication, évaluation de la qualité, discours organisationnel

Abstract: The imperative of organized communication compels service organizations to
develop coherent communication strategies that help create a consistent image of the organization.
The effectiveness of communication practices recommended by the organization is constantly
monitored using many evaluation mechanisms. Building on the example of an airline of North
American origin, this article analyzes the internal (performed by the services of quality control)
and external (conducted by customers) evaluation methods implemented within its call center.
Comparing the results of these two independent - but based on the same telephone conversation
- evaluations, can show either the difference of the interests of the company and its customers or
their distinct comprehension of the social goal of service interactions that is customer satisfaction. Keywords: service interactions, customer satisfaction, communication strategy, quality evaluation, organizational discourse 